\title{
A note on the effects of market inefficiency and portfolio constraints on the relationship between the expected return of an asset and the market
}

\author{
Thomas A. Severini* \\ Department of Statistics, Northwestern University, USA
}

Received: 23 August 2015

Revised: 9 December 2015

Accepted: 11 December 2015

\begin{abstract}
A key assumption of the Capital Asset Pricing Model is that the market portfolio is efficient; when it is inefficient, $\alpha$, the difference between the expected excess return of the asset and the value predicted by the CAPM, is non-zero. In this paper, a simple bound on $\alpha$ is given that depends on the efficiency of the market portfolio. Alternatively, the impact of inefficiency may be viewed in terms of its effect on $\beta$, the coefficient of the expected market return in the CAPM. A simple bound on the difference between $\beta$, based on an inefficient market portfolio, and $\beta$, based on an efficient portfolio, is also given. These results are used to assess the impact of portfolio constraints.
\end{abstract}

Keywords: alpha, asset pricing, beta, market efficiency

JEL Classification Codes: G12, C54

\section{Introduction}

How should we evaluate the sustainability of public debt? This is the issue that has been The set of results known collectively as the Capital Asset Pricing Model is one of the cornerstones of the mean-variance approach to portfolio theory. Generally speaking, the CAPM expresses the expected return on an asset as an affine combination of the expected return on an an efficient portfolio, often taken to be some form of a market portfolio, and the expected return of an asset that is uncorrelated with the efficient portfolio. It is well-known that the efficiency of the market portfolio is a necessary condition for the CAPM to hold (Roll, 1977; Ross, 1977).

Let $\alpha$ denote the difference between the expected return of an asset and the expected return predicted by the CAPM. When the CAPM holds, then $\alpha=0$ for all assets. The primary objective of this note is to consider the effect of market inefficiency on the relationship between the expected return on an asset and the expected return on the market portfolio. Two ways of measuring the impact of inefficiency are considered. One is its effect on $\alpha$; a simple bound for

\footnotetext{
*E-mail: severini@northwestern.edu.
}

Citation: Severini, T.A. (2015) A note on the effects of market inefficiency and portfolio constraints on the relationship between the expected return of an asset and the market, Economics and Business Letters, 4(4), 175-182. 
$\alpha$ is given that depends on the efficiency of the market portfolio. A second way to measure the impact of inefficiency is its effect on $\beta$, the coefficient of the return on the market portfolio in the CAPM. When the market portfolio inefficient, we can compare the value of $\beta$ to the value of $\beta^{-}$, the coefficient that would be obtained using an efficient portfolio; a simple bound for the difference $\mid \beta-\beta T$ is derived. These results are then applied to the case in which the portfolios under consideration are subject to constraints.

A number of authors have considered the effect of inefficiency on the relationship between the expected return of an asset and the market portfolio. Dybvig and Ross (1985) focuses on the conditions under which the expected return for a given asset lies above or below the security market line. Green (1986) considers the robustness of the linear relationship given by the CAPM under inefficiency of the market portfolio, similar to the analysis here, although from a different perspective. Diacogiannis and Feldman (2013) propose a pricing model based on an inefficient benchmark, including an adjustment for inefficiency. Markowitz (2005) discusses a number of issues related to market portfolio inefficiency and portfolio constraints.

\section{Basic framework and preliminary results}

Following Markowitz (1952), we model the return of an asset by a random variable, assumed to have finite mean and variance. For simplicity, we identify an asset by its return variable; e.g., we write "an asset $R$ " to denote an asset with return $R$, a random variable with a given mean and variance, denoted by $E(R)$ and $\operatorname{Var}(R)$, respectively. The covariance of assets $R_{1}$ and $R_{2}$ will be denoted by $\operatorname{Cov}\left(R_{1}, R_{2}\right)$.

Let $\mathcal{R}_{A}$ denote the set of all assets with finite mean and variance and let $\mathcal{R} \subset \mathcal{R}_{A}$ denote the set of assets under consideration. Assume that $\mathcal{R}$ is a convex set containing at least three assets with distinct means and that $\operatorname{Var}(R)>0$ for all $R \in \mathcal{R}$, except possibly for a unique risk-free asset. An asset $R$ is said to be in the interior of $\mathcal{R}$ if for any $R_{1} \in \mathcal{R}, t R_{1}+(1-t) R \in \mathcal{R}$ for all $|t|<\varepsilon$ for some $\varepsilon>0$.

Let $R^{*} \in \mathcal{R}$. We say that $R^{*}$ is efficient in $\mathcal{R}$ if for any asset $R \in \mathcal{R}$ such that $E(R)=$ $E\left(R^{*}\right), \operatorname{Var}(R) \geq \operatorname{Var}\left(R^{*}\right)$ and for any asset $R \in \mathcal{R}$ such that $E(R)>E\left(R^{*}\right), \operatorname{Var}(R)>$ $\operatorname{Var}\left(R^{*}\right)$.

The following proposition gives a general form of the CAPM and its converse; throughout the paper proofs are deferred until Section 7.

Proposition 1:

Let $R^{*}$ and $R_{0}$ be assets in $\mathcal{R}$ such that $\operatorname{Var}\left(R^{*}\right)>0, E\left(R^{*}\right) \neq E\left(R_{0}\right)$ and $\operatorname{Cov}\left(R^{*}, R_{0}\right)=0$.

For $R \in \mathcal{R}_{A}$, define $\beta(R)=\operatorname{Cov}\left(R, R^{*}\right) / \operatorname{Var}\left(R^{*}\right)$ and

$$
\alpha(R)=E(R)-E\left(R_{0}\right)-\beta(R)\left\{E\left(R^{*}\right)-E\left(R_{0}\right)\right\} .
$$

Let $\mathcal{R}_{0}=\{R \in \mathcal{R}: \alpha(R)=0\}$. Then $\mathcal{R}_{0}=\mathcal{R}$ if and only if the following conditions hold:

a. $R^{*}$ is in the interior of $\mathcal{R}$

b. $R^{*}$ is efficient in $\mathcal{R}$.

Proposition 1 may be used to establish many different versions of the CAPM. E.g., take $\mathcal{R}=$ $\mathcal{R}_{A}$ and $R_{0}=R_{f}$, the risk-free asset with $\operatorname{Var}\left(R_{f}\right)=0$; it follows that $R_{f}$ is uncorrelated with any other asset. We can take $R^{*}$ to be a portfolio that is efficient in $\mathcal{R}$, keeping in mind that $\mathcal{R}$ includes $R_{f}$; therefore, $R^{*}=R_{T}$, the tangency portfolio, assumed to be equivalent to the market portfolio, yielding the classical CAPM (Sharpe, 1964; Lintner, 1965; Mossin, 1966). If $\mathcal{R}$ consists only of risky assets, take $R^{*}$ to be any efficient portfolio and take $R_{0}$ to be the zero- $\beta$ 
portfolio with respect to $R^{*}$. Provided that $R^{*}$ is in the interior of $\mathcal{R}$ the CAPM holds (Black, 1972; Roll, 1977). If short sales on risky assets are not permitted, take $\mathcal{R}$ to be the set of portfolios with with non-negative weights on risky assets and take $R_{0}=R_{f}$, the risk-free asset. Since the market portfolio, with return $R_{M}$, is in the interior of $\mathcal{R}$, if $R_{M}$ is efficient, the CAPM holds with $R^{*}=R_{M}$. The case in which the efficient portfolio is not in the interior of $\mathcal{R}$ will be considered in Section 6.

The following corollary follows immediately from the proof of Proposition 1 . Recall that the affine hull of a set $A$ is the smallest affine set containing $A$. In the present context, the affine hull of a set of assets is the set of all portfolios contructed from those assets, allowing unlimited short sales.

Corollary 1:

If the CAPM holds for a set of assets $\mathcal{R}$, then it also holds for the affine hull of $\mathcal{R}$.

\section{The effect of an inefficient market portfolio}

Suppose that $R_{0}=R_{f}$, the risk-free asset. According to Proposition 1, a necessary condition for $\alpha(R)=0$ for all $R \in \mathcal{R}$ is for the portfolio $R^{*}$ to be efficient in $\mathcal{R}$. In this section, it is shown that if $R^{*}$ is nearly efficient, then $\alpha(R)$ is close to 0 for all $R \in \mathcal{R}$.

In practice, $\alpha$ and $\beta$ are generally estimated from data using least-squares regression. It is well-known that the standard error of the least-squares estimator of $\alpha$ is $\sigma_{\alpha} / \sqrt{n}$ where $\sigma_{\alpha}^{2}(R)=$ $\left(1+S R\left(R^{*}\right)^{2}\right)\left(1-\rho(R)^{2}\right) \operatorname{Var}(R), \rho(R)$ is the correlation of $R, R^{*}, S R(R)$ is the Sharpe ratio of an asset $R$, and $n$ is the sample size used in the estimation; see, e.g., Chapter 11 of Newbold, et al. (2013). Therefore, $\sigma_{\alpha}^{2}(R)$ is a measure of the information available regarding $\alpha(R)$,with a small value of $\sigma_{\alpha}^{2}(R)$ indicating that there is a relatively large amount of information about $\alpha(R)$.

Proposition 2:

Consider an affine set of assets $\mathcal{R}$. Suppose that $R^{*}$ is not necessarily efficient and let $\bar{R}^{*} \in \mathcal{R}$ be the minimum-variance portfolio with mean $E\left(R^{*}\right)$. Define $\phi=\operatorname{Var}\left(\bar{R}^{*}\right) /$ $\operatorname{Var}\left(R^{*}\right)$. Then, for $R \in \mathcal{R}$,

$$
\alpha(R)^{2} \leq \frac{1-\phi}{\phi} \frac{S R\left(R^{*}\right)^{2}}{1+S R\left(R^{*}\right)^{2}} \sigma_{\alpha}^{2}(R) \leq \frac{1-\phi}{\phi} \sigma_{\alpha}^{2}(R) .
$$

Therefore $\alpha(R)$ is small for all assets whenever the market portfolio is nearly efficient. When the market portfolio is not nearly efficient, $\alpha(R)$ is small for those assets for which $\sigma_{\alpha}(R)$ is small, e.g., those assets which are highly correlated with the market portfolio. This result is related to results of Jobson and Korkie (1982), who consider the extent to which it is possible to improve upon the market portfolio by changing the investment in a given asset; see also Grinold and Kahn (2000, Chap. 2) for related results in the context of active portfolio management.

The result in Proposition 2 measures the efficiency of $R^{*}$ relative to the minimum variance portfolio with the same mean as $R^{*}$. The following corollary shows that the same result holds if efficiency is measured relative to the portfolio with the maximum Sharpe ratio.

Corollary 2:

Let $R_{T}$ denote the element of $\mathcal{R}$ with the maximum Sharpe ratio and let $R \in \mathcal{R}$ satisfy $E(R)>E\left(R_{0}\right)$. Then Proposition 2 holds with $\phi$ defined as $S R\left(R^{*}\right)^{2} / S R\left(R_{T}\right)^{2}$. 


\section{Some empirical results}

To illustrate the relevance of Proposition 2 to the analysis of stock return data, the model $R-$ $R_{f}=\alpha+\beta\left(R_{m}-R_{f}\right)+\epsilon$, where $R$ is the return on a stock, $R_{m}$ is the return on a market index, $\epsilon$ is a mean-zero error term that is uncorrelated with $R_{m}, R_{f}$ is the risk-free rate of return, and $\alpha$ and $\beta$ are the parameters discussed above, was fit to the stocks represented in the Standard and Poor's 500 index as of November, 2015. The market index was taken to be the Wilshire 5000 Total Market Index and the risk-free rate was taken to be the 90-day U. S. Treasury Bill rate. Five years of monthly returns were analyzed, ending with November, 2015. Stocks in the S\&P 500 with returns for this period were used; there were 466 such stocks.

Least-squares estimates of $\alpha$ were computed for each stock. The mean value of these estimates for the stocks analyzed was 0.0031 , with a median value of 0.0035 ; the lower and upper quartiles were -0.0021 and 0.0092 , respectively. These values may be compared to the mean excess returns for the stocks, which had an average value of 0.0135 ; thus, the values of $\alpha$ makes a significant contribution to the mean returns of the stocks.

For each stock, an estimate of the ratio $\psi^{2}=\alpha(R)^{2} / \sigma_{\alpha}^{2}(R)$ was computed; according to Proposition 2, this ratio is bounded by $(1-\phi) / \phi$, where $\phi$ is a measure of the efficiency of the market portfolio. For the stocks considered, the maximum estimate of $\psi^{2}$ was 0.255 ; this result, along with the bound described above, leads to an estimated upper bound for $\phi$ of 0.797 . This result is similar to the empirical results of Haugen and Baker (1991) and of Clarke et al. (2006), which suggest that market portfolios have an efficiency in the range of 60 to $90 \%$.

To investigate the role of market inefficiency in the values of $\alpha$ a small simulation study was conducted for a specific model for the relationship between asset returns and those of an inefficient market portfolio. Using the notation of Proposition 2, suppose that $R^{*}=\bar{R}^{*}+\delta$ for a mean-zero random variable $\delta$ that is uncorrelated with $\bar{R}^{*}$. Write $R-R_{f}=\bar{\beta}\left(\bar{R}^{*}-R_{f}\right)+$ $\epsilon$ for the equation relating the return $R$ on a given asset to the return on the efficient portfolio, where $R_{f}$ denotes the return on the risk-free asset and $\epsilon$ denotes a mean-zero random variable that is uncorrelated with $\bar{R}^{*}$ and with $\delta$. Thus, $\bar{\beta}$ denotes the value of beta with respect to an efficient portfolio; in this model the value of $\alpha$ is taken to be zero, consistent with the CAPM.

Data were simulated from this model for the case in which $\bar{R}^{*}-R_{f}$ has expected value 0.01 and standard deviation 0.025; these are based on the observed values for the Wilshire 5000 index, taking into account its inefficiency. The standard deviation of $R-R_{f}$ was taken to 0.06 , roughly the median value for the stocks in the S\&P 500. Different values of $\bar{\beta}$ and $\phi$, the efficiency of the market portfolio were considered. Sixty returns for each asset were drawn from normal distributions and the returns were taken to be serially uncorrelated. Thus, the simulated data values may be viewed as simulated values of five years of monthly returns; this procedure was repeated 10000 times.

For $\bar{\beta}=1$ and $\phi=0.8$, the average estimate of $\alpha$ was roughly 0.0020 , slightly smaller than the observed average value for the S\&P 500 stocks. As expected, for larger values of $\phi$, the average value of $\alpha$ was smaller while for smaller $\phi$ it was larger; for instance, for $\phi=0.9$, the average $\alpha$ was 0.0009 and for $\phi=0.7$, the average $\alpha$ was 0.0030 . For fixed $\phi$, values of $\alpha$ tend to increase with $\bar{\beta}$; for instance using $\phi=0.8$, for $\bar{\beta}=1.5$ the average $\alpha$ was 0.0029 , while for $\bar{\beta}=0.5$, it was 0.00098 . In interpreting this result, it is important to keep in mind that $\bar{\beta}$ is the value of beta with respect to the unobserved efficient market portfolio. 


\section{The effect of inefficiency on $\beta$}

The result in Proposition 2 expresses the effect of inefficiency of the market portfolio in terms of the value of $\alpha$. Alternatively, we may view the effect of inefficiency on $\beta$ : if $R^{*}$ is inefficient, we are using the wrong portfolio to compute $\beta$, in a certain sense.

As in Proposition 2 , let $R^{*}$ denote the portfolio upon which the CAPM is based and let $\bar{R}^{*}$ denote the efficient portfolio with the same mean as $R^{*}$. Let $\bar{\beta}(R)=\operatorname{Cov}\left(R, \bar{R}^{*}\right) / \operatorname{Var}\left(\bar{R}^{*}\right)$ denote the value of $\beta$ for an asset $R$ based on the efficient portfolio $\bar{R}^{*}$. Let $\sigma_{\beta} / \sqrt{n}$ denote the standard error of the least-squares estimator of $\beta$; then $\sigma_{\beta}^{2}(R)=\left(1-\rho(R)^{2}\right) \operatorname{Var}(R) /$ $\operatorname{Var}\left(R^{*}\right)$; see, e.g., Chapter 11 of Newbold et al. (2013). As with $\sigma_{\alpha}^{2}(R)$, a small value of $\sigma_{\beta}^{2}(R)$ indicates that there is a relatively large amount of information available regarding $\beta(R)$.

The following result gives a bound on the difference between $\beta(R)$ and $\bar{\beta}(R)$.

Proposition 3:

Consider an affine set of assets $\mathcal{R}$. Suppose that $R^{*}$ is not necessarily efficient and let $\bar{R}^{*} \in \mathcal{R}$ be the portfolio in $\mathcal{R}$ with mean $E\left(R^{*}\right)$ with minimum variance. Define $\phi=$ $\operatorname{Var}\left(\bar{R}^{*}\right) / \operatorname{Var}\left(R^{*}\right)$. Then, for $R \in \mathcal{R}$,

$$
[\beta(R)-\bar{\beta}(R)]^{2} \leq \frac{1-\phi}{\phi} \sigma_{\beta}^{2}(R) .
$$

\section{The effect of portfolio constraints}

Let $\mathcal{R}$ be a set of portfolios subject to some constraints such that $\mathcal{R}$ is not affine. Let $R^{*}$ be efficient in $\mathcal{R}$ and take $R_{0}=R_{f}$, the risk-free asset. According to Proposition 1, if $R^{*}$ is in the interior of $\mathcal{R}$ then the CAPM holds for all $R \in \mathcal{R}$. However, when portfolios are required to satisfy certain constraints, it is not uncommon for the efficient portfolios to lie on the boundary of $\mathcal{R}$, on which the one or more of the constraints is active. Therefore, in the remainder of this section, we assume that $R^{*}$ lies on the boundary of $\mathcal{R}$. The following result shows that, in this case, it is the affine hull of $\mathcal{R}$ that is relevant to the CAPM rather than $\mathcal{R}$ itself.

\section{Proposition 4:}

Assume that $\mathcal{R}$ is not affine and that $R^{*}$ lies on the boundary of $\mathcal{R}$. Then the $\alpha(R)=0$ for $R \in \mathcal{R}$ if and only if $R^{*}$ is efficient in $\overline{\mathcal{R}}$, the affine hull of $\mathcal{R}$.

Now suppose that $R^{*}$ is not efficient in $\overline{\mathcal{R}}$ and let $\bar{R}^{*}$ be an efficient portfolio in $\overline{\mathcal{R}}$ with the same expected value as $R^{*}$. Then the CAPM holds for $\overline{\mathcal{R}}$, and hence for $\mathcal{R}$, using $\bar{R}^{*}$ as the efficient portfolio. Therefore, using $R^{*}$, the efficient portfolio subject to constraints, is a case of using an inefficient portfolio, as discussed in the previous section. Therefore, the bound (2) holds so that, for $R \in \mathcal{R}$,

$$
\alpha(R)^{2} \leq \frac{1-\phi}{\phi} \sigma_{\alpha}^{2}
$$

with $\phi$ interpreted as the efficiency of $R^{*}$ relative to $\bar{R}^{*}$.

That is, the impact of constraints on the CAPM may be viewed in terms of the ratio of the variance of the unconstrained efficient portfolio to the variance of the constrained efficient portfolio. If this ratio is close to 1, i.e., if the variance of the constrained efficient portfolio is close to the variance of the unconstrained efficient portfolio, then the constraints have only a minor impact on the CAPM. On the other hand, if the variance of the unconstrained efficient portfolio is small relative to the variance of the constrained portfolio, that is, if imposing 
constraints greatly increases the variance of the efficient portfolio, then the values of $\alpha(R)$ for the constrained portfolios may be relatively large.

\section{Technical details}

\section{Proof of Proposition 1:}

First assume that conditions (a) and (b) hold. Fix $R \in \mathcal{R}$ and let $\hat{R}=\theta R^{*}+(1-\theta) R_{0}, \theta=$ $\left\{E(R)-E\left(R_{0}\right)\right\} /\left\{E\left(R^{*}\right)-E\left(R_{0}\right)\right\}$. Note that $E(\hat{R})=E(R)$,

$$
\alpha(R)=\{\theta-\beta(R)\}\left\{E\left(R^{*}\right)-E\left(R_{0}\right)\right\},
$$

and, since $\operatorname{Cov}\left(R^{*}, R_{0}\right)=0$,

$$
\operatorname{Cov}\left(\widehat{R}, R^{*}\right)=\theta \operatorname{Var}\left(R^{*}\right)
$$

For $t \in \Re$, define $R_{t}=R^{*}+t(R-\hat{R})$. Note that $R_{t}=R_{1 t} / 2+R_{2 t} / 2$ where $R_{1 t}=(1-$ $2 t(\theta-1)) R^{*}+2 t(\theta-1) R_{0}$ and $R_{2 t}=(1-2 t) R^{*}+2 t R$. By condition (a), $R_{1 t}$ and $R_{2 t}$ are in $\mathcal{R}$ for sufficiently small $|t|$ and, hence, $R_{t} \in \mathcal{R}$. Since $E\left(R_{t}\right)=E\left(R^{*}\right)$, condition (b) implies that $\operatorname{Var}\left(R_{t}\right) \geq \operatorname{Var}\left(R^{*}\right)$ for all $|t|<\varepsilon$ for some $\varepsilon>0$.

Suppose that $0<t<\varepsilon$. Then we must have $t \operatorname{Var}(R-\hat{R})+2 \operatorname{Cov}\left(R^{*}, R-\hat{R}\right) \geq 0$ for all $0<t<\varepsilon$. It follows $\operatorname{Cov}\left(R^{*}, R-\hat{R}\right) \geq 0$. A similar argument for $-\varepsilon<t<0$ shows that $\operatorname{Cov}\left(R^{*}, R-\hat{R}\right) \leq 0$. Hence, $\operatorname{Cov}\left(R^{*}, R-\hat{R}\right)=0$ and, by (6), $\theta=\beta(R)$; it follows from (5) that $\alpha(R)=0$ so that $\mathcal{R}_{0}=\mathcal{R}$.

Now assume that $\mathcal{R}_{0}=\mathcal{R}$ so that $\alpha(R)=0$ for $R \in \mathcal{R}$. Note that $\alpha(\cdot)$ is a linear function on $\mathcal{R}_{A}$ and that $\alpha\left(R^{*}\right)=0$. Let $R$ be an asset such that $\alpha(R)=0$. Then, for any $t \in \Re$,

$$
\alpha\left((1-t) R^{*}+t R\right)=(1-t) \alpha\left(R^{*}\right)+t \alpha(R)=0 .
$$

It follows that (a) holds.

To establish (b), let $R_{1}$ be such that $\alpha\left(R_{1}\right)=0$ and $E\left(R_{1}\right)>E\left(R^{*}\right)$. Then

$$
\frac{\operatorname{Cov}\left(R^{*}, R_{1}\right)}{\operatorname{Var}\left(R^{*}\right)}\left\{E\left(R^{*}\right)-E\left(R_{0}\right)\right\}=E\left(R_{1}\right)-E\left(R_{0}\right)>E\left(R^{*}\right)-E\left(R_{0}\right) .
$$

Suppose that $E\left(R^{*}\right)>E\left(R_{0}\right)$. By $(8), \operatorname{Cov}\left(R^{*}, R_{1}\right)>\operatorname{Var}\left(R^{*}\right)$, that is, $\operatorname{Cov}\left(R^{*}, R_{1}-\right.$ $\left.R^{*}\right)>0$; hence, $\operatorname{Var}\left(R_{1}\right)>\operatorname{Var}\left(R^{*}\right)+\operatorname{Var}\left(R_{1}-R^{*}\right) \geq \operatorname{Var}\left(R^{*}\right)$.

If $E\left(R^{*}\right)<E\left(R_{0}\right)$ then $\operatorname{Cov}\left(R^{*}, R_{1}\right)<-\operatorname{Var}\left(R^{*}\right)$ and applying the same argument to $\operatorname{Var}\left(R_{1}\right)=\operatorname{Var}\left[R^{*}-\left(R^{*}+R_{1}\right)\right]$ shows that $\operatorname{Var}\left(R_{1}\right) \geq \operatorname{Var}\left(R^{*}\right)+\operatorname{Var}\left(R^{*}+R_{1}\right)$. Therefore, $E\left(R_{1}\right)>E\left(R^{*}\right)$ implies that $\operatorname{Var}\left(R_{1}\right)>\operatorname{Var}\left(R^{*}\right)$.

If $E\left(R_{1}\right)=E\left(R^{*}\right)$ a simpler version of this argument applies: then $\operatorname{Cov}\left(R^{*}, R_{1}-R^{*}\right)=$ 0 and, hence, $\operatorname{Var}\left(R_{1}\right) \geq \operatorname{Var}\left(R^{*}\right)$. It follows that $R^{*}$ is efficient in $\mathcal{R}_{0}$, proving the result.

\section{Proof of Proposition 2:}

Fix an asset $R$ and define $\theta, \hat{R}$ and $R_{t}$ as in the proof of Proposition 1; for convenience, write $\beta$ for $\beta(R), \alpha$ for $\alpha(R)$, and so on.

Since $R_{t} \in \mathcal{R}$ and $E\left(R_{t}\right)=E\left(R^{*}\right), \operatorname{Var}\left(R_{t}\right) \geq \operatorname{Var}\left(\bar{R}^{*}\right)=\phi \operatorname{Var}\left(R^{*}\right)$ for all $t$. Hence,

$$
\inf _{t} \operatorname{Var}\left(R_{t}\right)=\operatorname{Var}\left(R^{*}\right)-\frac{\operatorname{Cov}\left(R^{*}, R-\hat{R}\right)^{2}}{\operatorname{Var}(R-\hat{R})} \geq \phi \operatorname{Var}\left(R^{*}\right) ;
$$

it follows that

$$
\frac{\operatorname{Cov}\left(R^{*}, R-\hat{R}\right)^{2}}{\operatorname{Var}(R-\hat{R}) \operatorname{Var}\left(R^{*}\right)} \leq 1-\phi
$$


Note that $\operatorname{Cov}\left(R^{*}, R-\hat{R}\right)=(\beta-\theta) \operatorname{Var}\left(R^{*}\right)$; therefore, by (10),

$$
|\beta-\theta|^{2} \leq(1-\phi) \frac{\operatorname{Var}(R-\hat{R})}{\operatorname{Var}\left(R^{*}\right)} .
$$

Writing $R-\hat{R}=R-\theta R^{*}-(1-\theta) R_{0}=R-\beta R^{*}+(\beta-\theta) R^{*}+(\theta-1) R_{f}$,

$$
\operatorname{Var}(R-\hat{R})=\operatorname{Var}\left(R-\beta R^{*}\right)+(\beta-\theta)^{2} \operatorname{Var}\left(R^{*}\right) .
$$

It follows from (11) that

$$
(\beta-\theta)^{2} \leq \frac{1-\phi}{\phi} \frac{\operatorname{Var}\left(R-\beta R^{*}\right)}{\operatorname{Var}\left(R^{*}\right)}
$$

From the definitions of $\beta$ and $\theta$,

$$
\operatorname{Var}\left(R-\beta R^{*}\right)=\frac{\sigma_{\alpha}^{2}}{1+S R\left(R^{*}\right)^{2}} \quad \text { and } \quad(\theta-\beta)^{2}=\frac{\alpha}{\left[E\left(R^{*}\right)-E\left(R_{0}\right)\right]^{2}} .
$$

It follows that

$$
\alpha^{2} \leq \frac{1-\phi}{\phi} \frac{\sigma_{\alpha}^{2}}{1+S R\left(R^{*}\right)^{2}} S R\left(R^{*}\right)^{2} .
$$

The result now follows from the fact that $S R\left(R^{*}\right)^{2} /\left[1+S R\left(R^{*}\right)^{2}\right] \leq 1$.

\section{Proof of Corollary 2:}

Define $R_{t}$ as in the proof of Proposition 2. Then $S R\left(R_{t}\right)^{2} \leq S R\left(R_{T}\right)^{2}=S R\left(R^{*}\right)^{2} / \phi$. Since $R_{t}$ and $R^{*}$ have the same mean, this implies that $\operatorname{Var}\left(R_{t}\right) \geq \phi \operatorname{Var}\left(R^{*}\right)$ for all $t$. The remainder of the proof follows as above.

\section{Proof of Proposition 3:}

Define $\alpha(R)$ as in Proposition 1. Since

$$
\alpha(R)=[\bar{\beta}(R)-\beta(R)]\left[E\left(R^{*}\right)-E\left(R_{0}\right)\right],
$$

it follows from (15) that

$$
[\bar{\beta}(R)-\beta(R)]^{2} \leq \frac{1-\phi}{\phi} \frac{\sigma_{\alpha}^{2}}{1+S R\left(R^{*}\right)^{2}} \frac{S R\left(R^{*}\right)^{2}}{\left[E\left(R^{*}\right)-E\left(R_{0}\right)\right]^{2}} .
$$

Using the fact that

$$
\begin{gathered}
\sigma_{\alpha}^{2}(R)=\left(1+S R\left(R^{*}\right)^{2}\right) \operatorname{Var}\left(R^{*}\right) \sigma_{\beta}^{2}(R), \\
{[\bar{\beta}(R)-\beta(R)]^{2} \leq \frac{1-\phi}{\phi} \sigma_{\beta}^{2}(R) \frac{\operatorname{Var}\left(R^{*}\right) S R\left(R^{*}\right)^{2}}{\left[E\left(R^{*}\right)-E\left(R_{0}\right)\right]^{2}}=\frac{1-\phi}{\phi} \sigma_{\beta}^{2}(R),}
\end{gathered}
$$

proving the result.

\section{Proof of Proposition 4:}

Suppose that $R^{*}$ is efficient in $\overline{\mathcal{R}}$. Then, by Proposition 1, the CAPM holds for $\overline{\mathcal{R}}$ and, hence, it holds for $\mathcal{R}$.

Conversely, suppose that, even though $R^{*}$ is on the boundary of $\mathcal{R}$, the CAPM still holds for $\mathcal{R}$; then, by Corollary 1 , the CAPM holds for $\overline{\mathcal{R}}$ and, hence, by Proposition $1, R^{*}$ is efficient in $\overline{\mathcal{R}}$. The result follows. 


\section{References}

Black, F. (1972) Capital Market Equilibrium with Restricted Borrowing, Journal of Business, 45, 444-455.

Clarke, R., de Silva, H. and Thorley, S. (2006) Minimum-Variance Portfolios in the U. S. Equity Market, Journal of Portfolio Management, 33, 1-14.

Diacogiannis, G. and Feldman, D. (2013) Linear Beta Pricing with Inefficient Benchmarks, Quarterly Journal of Finance, 3, 1350004-1 - 135004-35.

Dybvig, P.H. and Ross, S.A. (1985) The Analytics of Performance Measurement Using a Security Market Line, Journal of Finance, 40, 401-416.

Green, R.C. (1986) Benchmark Portfolio Inefficiency and Deviations from the Security Market Line . Journal of Finance, 41, 295-312.

Grinold, R.C. and Kahn, R.N. (2000) Active Portfolio Management. McGraw-Hill, New York, $\mathrm{NY}, 2^{\text {nd }}$. ed.

Haugen, R.A. and Baker, N.L. (1991) The efficient market inefficiency of capitalizationweighted stock portfolios, Journal of Portfolio Management, 17, 35-40.

Jobson, J.D. and Korkie, B. (1982) Potential Performance and Tests of Portfolio Efficiency, Journal of Financial Economics, 10, 433-466.

Lintner, J. (1965) The valuation of risk assets and the selection of risky investments in stock portfolios and capital budget, Review of Economics \& Statistics, 47, 13-37.

Markowitz, H. (1952) Portfolio selection, Journal of Finance, 7, 77-91.

Markowitz, H.M. (2005) Market Efficiency: A Theoretical Distinction and So What?, Financial Analysts Journal, 61, 17-30.

Mossin, J. (1966). Equlibrium in a capital asset market. Econometrica, 34, 768-783.

Newbold, P. and Carlson, W.L. and Thorne, B.M. (2013) Statistics for Business and Economics, Pearson, Upper Saddle River NJ, $8^{\text {th }}$ ed.

Roll, R.A. (1977) critique of the asset pricing theory's tests: part I: on past and potential testability of the theory, Journal of Financial Economics, 4, 129-176.

Ross, S.A. (1977) The Capital Asset Pricing Model (CAPM), Short-Sale Restrictions and Related Issues, Journal of Finance, 32, 177-183.

Sharpe, W.F. (1964) Capital asset prices: a theory of market equilibrium under conditions of risk, Journal of Finance, 19, 425-442. 